作组织, 具体部署, 以及为实现这一规划所应 采取的各项措施等问题,进行了反复研究,取 得了较为一致的意见。

这次基本粒子座谈会是我国关于基本粒 子理论研究工作的一次重要的会议. 代表们 表示,一定要把党的十一大的公报精神化为
巨大的动力, 最紧密地团结在以华主席为首 的党中央周围, 以辩证唯物主义为指导, 发扬 不畏艰险, 勇于攀登的精神,以新的面貌和新 的成就迎接全国科学大会的召开, 为把我国 建成为伟大的社会主义现代化强国而努力奋 斗.

\title{
向癌 症进军的新起点
}

\section{一一全国肪痹会议在北京胜利召开}

在华主席、党中央关怀下,卫生部于 1977 年6月18日至 7 月 2 日在北京召开了全国肿 瘤防治研究工作会议. 会议总结交流了走我 国自己肿㾇防治研究道路所取得的成绩和经 验,制订了肿瘤防治研究规划.

我国劳动人民有着几千年与癌症作斗争 的历史. 但我国关于癌症的现代研究解放后 在毛主席、周总理的亲切关怀下才逐步开展 起来. 1958 年, 特别是无产阶级文化大革命 以来, 肿㾇防治研究工作有了迅速的发展.

几年来, 我国从中央到地方正在建立健 全各级肿瘤防治研究机构; 不少地区,尤其是 一些农村、工矿高发区防治点和一些城市街 道建立了较完善的防癌网：我国一些重点肿 痹的农村、工矿高发区防治点继林县食管癌 防治点之后逐步地建立起来, 在抓“三早”(早 期发现、早期诊断、早期治疗)、政“三关”(病 因、诊断、根治) 方面做了不少工作; 各种全 国性和地区性的协作组织不断发展; 由各级 专业人员和赤脚医生、红医工及基层医务人 员组成的防治研究大军茁壮成长; 许多非肿 瘤专业以至非医学的有关科研部门和单位也 参加到肿瘤防治研究工作中来. 流行病学调 查研究工作广泛开展, 进展迅速. 我国仅用 3-5 年的时间即可基本摸清全国肿瘤的发 病情况和分布特点, 为研究发病原因和有计
划地开展防治工作提供了大量科学数据.

我国的肿㿔诊断技术几年来有了较大的 改善和提高.一些重点肿瘤如食管癌、肝癌、 鼻咽癌、宫颈癌等的早期诊断水平显著提高, 已能从自党症状的病人身上发现很早期的肝 癌、食管癌、肺癌等. 通过普查发现的癌病 人，早期癌比例可达 $80 \%$ 以上，使许多病 人可以获得早期诊断和早期治序. 应用我国 自己创造的食管“拉网”细胞学方法,食管癌 阳性率可达 $90 \%$ ，并可通过分段拉网进行定 位; 改进拉网法, 还提高了贵门癌细胞学诊 断的阳性率，为贵门癌的早期诊断做出了贡 献; 通过大量实践, 逐步摸清肝癌甲胎蛋白 (AFP) 含量动态变化的一些规律, 建立简便 易行、灵敏度高的测定方法（反向血球凝集 法、晶格参入放射免疫火箭自显影法)，使甲 胎蛋白测定成为我国肝癌早期诊断的有效方 法, 并可用于农村大面积普查, 发现了许多早 期无自觉症状的肝癌病人; 通过不断改进脱 落细胞采集方法, 使鼻咽癌脱落细胞学的阳. 性诊断率达到 $90 \%$ 以上. 几年来,细胞学和 免疫学的一些诊断方法已在我国广泛应用, 在一些高发区正在普及到赤脚医生、红医工 用于普查. 此外, 癌症诊断的不少新技术、新 方法、新仪器我国也已开始应用.

几年来,不少地区,特别是一些防治试点 
开展了大量的预防工作, 通过普查抓“三早”, 使许多病人获得早期诊断和早期治疗，提高 了治愈率; 对与癌症发生有关的一些疾病和 病变,如一些部位的溃疡、糜烂、炎症、增生、 息肉和癌前病变,进行普查普治,有的已收到 较好效果, 有利于降低肿瘤的发病率。1969 年以来, 上海市重视对官颈炎症和癌前病变 的治疗，使官颈癌的发病率明显下降. 上海 市纺织系统自 1958 年以来,坚持开展官颈癌 及有关疾病和癌前病变的普查普治工作，根 据至 1972 年底的统计,女工宫颈癌的标准化 患病率和发病率, 1972 年较 1958 年分别下 降 $81 \%$ 和 $81.4 \%$; 许多防治点还针对主要病 因线索,结合农村、工矿卫生革命，爱国卫生 运动，妇幼保健等采取了多方面的群众性的 预防措施，努力降低发病率。有关某些致癌 因素的有效去除方法的研究也取得了一定的 进展。

由于普查、早期发现、中西医结合治疗， 我国一些肿㾇如食管癌、鼻咽癌、肝癌、官颈 癌、乳腺癌、线癌等的治愈率显著提高, 河南 林县人民医院食管癌总的术后五年生存率已 达 $46 \% ， 170$ 例早期食管癌术后五年生存率 达 $90.3 \%$ ，其中 93 人随访 8 年无复发，大部 分参加了生产劳动; 广东省中山医学院肿瘤 医院鼻咽癌总的治后五年生存率达 49.5\%; 据某市综合统计，肝癌治后五年生存率达 $8.2 \%$, 有一个防治点 9 例早期肝癌术后至今 已三年余, 5 例生存尚好,并不同程度地恢复 了劳动力; 官颈瘄的中西医治愈水平普遍提 高, 上海市纺织系统由于坚持普查普治,女工 官颈癌总的五年、十年、十五年生存率分别已 达 $95.7 \% 、 92.4 \% 、 90.6 \%$ ；一些单位宫颈癌 I 期治愈率已可达 $95 \%$ 以上以至 $100 \%$; 䄽 癌中西医结合治疗效果不断提高, 一些单位 I 期乳癌术后配合或不配合其他治疗，五年 生存率可达 $90 \%$ 以上; 线癌大剂量间歇化疗 加用中草药,死亡率已下降到 $29.3 \%$.
在抗癌药物和仪器设备的研制方面也取 得了较快的进展. 我国除已能生产出国际上 常用的几十种有效药物之外, 还自行设计研 制了十几种有效的化学药物, 并通过实验和 临床研究, 从中草药中找到一些疗效较好的 药物, 目前抗癌中草药运动正在广泛开展。 在抗癌药篮选方法上也进行了研究, 创造了 精原细胞法等方法. 诊断治疗肿瘤用的一些 较先进或较大型的仪器设备, 包括多探头同 位素彩色扫描仪, 闪烁照相机、直线加速器、 模拟定位机等,我国基本上也能生产.

在基础理论方面,这几年不断排除“四人 帮”的干扰和破坏，逐步开展肿㿔病因学、发 病学、免疫学等方面的研究, 取得了一定的进 展. 近年来, 在流行病学调查的基础上, 对我 国的一些重点肿㾇开展了不同程度的病因调 查和实验研究，特别是对食管癌、肝癌等进行 了多学科、多部门的病因综合考察,获得了大 量第一手资料,在此基础上密切结合实际, 有 针对性地进行实验研究, 对一些致癌因素有 了较深入的了解,并有一些新的发现,从而掌 握了一些肿瘤的主要病因线索. 现在对病毒 病因、化学病因、霉菌病因及遗传病因的研究 正在深人. 人食管癌上皮细胞株、人肝癌细 胞株、人白血病细胞株, 特别是人鼻咽癌上皮 细胞株的培养成功, 多种化学物质多途径诱 发动物㐭咽癌模型、病毒诱发动物白血病模 型、可移植性动物乳癌模型等的建立, 为病因 学、发病学、药物篮选、实验治疗以及癌细胞 生物特性等基础方面的研究提供了有用的工 具. 关于中西医结合的某些基础理论方面的 研究也正在开展.

这次会议是我国向癌症进军的新起点. 大家决心紧跟华主席, 抓纲治国, 大干快上, 为早日攻克㾂症, 攀登医学科学高峰, 对人类 做出贡献而努力.

[王汝宽] 